\title{
ESSAI DE CLASSIFICATION DES NÉMATODES ACUARIIDAE
}

\author{
Par Alain G. CHABAUD et Annie J. PETTER
}

\section{I. - INTRODUCTION}

La famille des Acuariidx a une systématique spécialement intéressante, car le recoupement d'éléments nombreux et d'origines diverses permet d'établir une sériation phylogénique relativement très précise.

Trois sortes de caractères peuvent être utilisés :

1) Morphologie de l'adulte.

L'anatomie générale des adultes, et en particulier la structure génitale des femelles, bien étudiée par Seurat, a permis depuis longtemps d'avoir une idée sur la phylogénie du groupe et de savoir que les espèces à cordons simples sont plus primitives que les espèces à cordons complexes.

2) Vitesse du développement chez l'hôte intermédiaire.

Nous avons cherché à démontrer (1949) que les Spirurides plus primitifs ont une évolution plus longue chez l'hôte intermédiaire. La classification qui peut être obtenue avec les chiffres actuellement connus coïncide avec les données phylogéniques obtenues par l'étude des adultes.

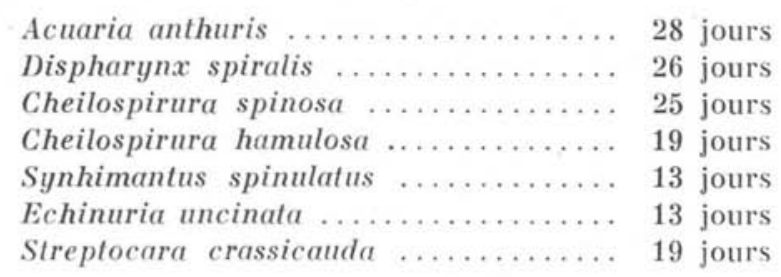

3) Morphologie des larves.

Seurat (1919), Chabaud (1954) et Osche (1955) ont insisté sur le curieux phénomène de « récapitulation ontogénique » qui fait que, 
dans certains cas, les larves des espèces spécialisées revêtent la morphologie des adultes d'espèces plus primitives. Les exemples connus sont :

Dispharynx spiralis qui, au $3^{\circ}$ et $4^{\circ}$ stades, a le type Acuaria.

Synhimantus laticeps et $S$. robertdollfnsi qui ont, au $3^{\circ}$ stade, le type Acuaria.

Synhimantus (Desportesius) spinulatus qui, au $3^{\circ}$ stade, a le type Acuaria, et au $4^{e}$ stade le type Syncuaria.

Cosmocephalus obvelatus qui a, au $4^{e}$ stade, le type Synhimantus.

Enfin Stammerinema soricis qui a, au $3^{e}$ stade, un type proche de Paracuaria et d'Acuaria quant aux cordons, et proche d'Echinuria quant aux épines latérales.

Les bases phylogéniques du groupe sont donc admises par tous les auteurs, et personne ne conteste que les formes à cordons complexes dérivent des formes à cordons simples. Cependant, si l'on ne considère que la forme des cordons, l'on peut imaginer plusieurs filiations possibles. Il est bien difficile, par exemple, de savoir quels sont les rapports entre le genre Rusguniella à cordons courts et le genre Syncuaria qui lui est homologue, mais a des cordons longs. Les classifications proposées sont donc assez différentes les unes des autres. Nous passerons en revue les trois types principaux de classifications actuellement proposées en indiquant quels sont les points qui nous paraissent sujets à critiques.

\section{II. - CLASSIFICATIONS ACTUELLES}

\section{$1^{\circ}$ Classification de Chitwood et Wehr (1934).}

Nous avons, en collaboration avec R.-Ph. Dollfus (1957), déjà donné les principales objections concernant ce système. Nous avons insisté sur la nécessité qu'il y avait à ne pas définir la famille uniquement sur les caractères cuticulaires, pour éviter toute confusion avec certains Habronematinæ tels que Parabronema, Hadjelia et Histiocephalus. Les objections que nous voudrions soulever aujourd'hui concernent la définition et la composition de la sousfamille des Seuratiinæ.

En dehors d'éléments communs à tous les Acuariidæ, la sousfamille est caractérisée essentiellement par le texte suivant : «Head appendages not in form of ribbon-like cordons or bands running longitudinally on body, but in form of a collarette surrounding head at base of pseudolabia ; free margin of collarette denticulated. 
Deirids (cervical papillae) variable in form. Type genus. - Seuratia Skrjabin, 1916. » La sous-famille comprend les trois genres Streptocara, Seuratia et Yseria.

a) Genre Yseria.

Le genre Yseria fut créé par Gedoelst (1919) avec comme espèce type $Y$. californica, mais, dès 1922, Gedoelst et Liégeois ont reconnu que l'espèce était en réalité un Streptocara, proche de l'espèce type $S$. pectinifera, et ils ont mis le genre en synonymie. Cram (1927) et Wehr (1934) semblent ne pas avoir eu connaissance de la publication de 1922, et ont pris le nom d'Yseria pour désigner le Spiroptera coronata Molin 1860. Cette seconde espèce est très différente des Streptocara, mais le nom d'Yseria ne peut être repris, puisque l'espèce type d'Yseria est un Streptocara.

Il semble a priori qu'il faille créer un nouveau genre pour placer Spiroptera coronata, ainsi qu'une autre espèce nommée Yseria quadripartita par Clapham (1945). Nous ne pensons pas cependant que ce nouveau genre puisse rester groupé avec Streptocara et Seuratia, car l'ornementation céphalique n'a pas la forme d'une collerette entourant la base des pseudo-lèvres, mais plutôt la forme de lames divisées à l'apex, ce qui place le genre dans les Schistorophinæ; et nous ne voyons plus alors aucun élément qui nécessite la création d'un nouveau genre, car rien n'oppose formellement coronota et quadripartita aux espèces du genre Ancyracanthopsis.

Nous pensons done en conclusion que le genre Yseria Gedoelst disparaît automatiquement, comme l'avait indiqué l'auteur luimême, puisque l'espèce type est en réalité un Streptocara. En outre, il nous semble inutile de créer un autre genre pour classer les espèces qui avaient été rangées sous le nom d'Yseria, car elles appartiennent en réalité aux Schistorophinæ et peuvent entrer dans le cadre de l'ancien genre Ancyracanthopsis.

\section{b) Sous-famille des Seuratiinae.}

La sous-famille ne comprend donc plus que les deux genres Streptocara et Seuratia, ce qui semble à première vue ne pas pouvoir être maintenu, car les deux genres sont très différents l'un de l'autre.

Les auteurs russes ont rapproché Streptocara des genres Rusguniella et Aviculariella, alors que Osche rapproche ces deux genres Rusguniella et Aviculariella de Seuratia.

L'étude que nous avons faite récemment (Petter, 1959) nous a précisément conduits à rapprocher tous ces genres et nous considérons Streptocara et Seuratia comme les deux points extrêmes 
d'une même série évolutive. Nous donnerons plus bas de nouveaux arguments à l'appui de cette thèse et pensons donc qu'il est possible de conserver la sous-famille des Seuratiinæx à condition de modifier sa définition et d'y inclure les nombreux genres qui relient Streptocara et Seuratia.

\section{$2^{\circ}$ Glassification des auteurs soviétiques.}

Les auteurs soviétiques: Skrjabin (1941), Sobolev (1949), Guschanskaja (1951), ont modifié la nomenclature admise depuis Chitwood et Wehr.

Il n'y a plus une famille et trois sous-familles, mais trois familles et douze sous-familles.

Les modifications semblent avoir pour but de rendre les déterminations plus faciles, car les auteurs se basent essentiellement sur les ornementations cuticulaires, et forment ainsi des familles ou des sous-familles, qui ont pour seul élément commun tel ou tel caractère cuticulaire, facilement appréciable.

L'Opredelitel forme ainsi un livre utile, qui semble facile à manier pour les chercheurs peu spécialisés en Nématologie.

Nous ne voulons pas cependant suivre cette systématique, car elle nous semble marquer un recul vis-à-vis des connaissances acquises depuis plus de 30 ans. Les affinités naturelles des différents genres sont négligées aux dépens des caractères cuticulaires qui sont précisément les éléments les plus sensibles aux phénomènes de convergence.

Il nous semble impossible de placer presque tous les Habronèmes à cuticule ornée (famille des Histiocephalidæ) parmi les « Acuarioidea $»$.

Des genres tels que Cyclozone ou Ancyracanthus n'ont, en dehors d'une vague similitude dans les dessins cuticulaires, aucun rapport avec les Acuariidæ.

La sous-famille des Echinuriinæ est basée sur l'existence d'épines sur les lignes latérales. Pour nous, les genres qui y sont inclus, tels que Seuratia et Echinuria, appartiennent à des phylums indépendants, et le seul point qu'ils ont en commun est d'être des espèces hyperévoluées (cf. Osche, 1955), ce qui entraîne fréquemment un fort développement des épines.

Il nous semble donc que les principales modifications apportées par l'Opredelitel dans la famille des Acuariidæ ne peuvent pas être conservées. 


\section{$3^{\circ}$ Glassification de Osche (1955).}

La principale modification apportée par Osche, par rapport à la classification de Chitwood et Wehr, a été de rapprocher le genre Seuratia de Rusguniella et d'Aviculariella. La sous-famille des Seuratiinæ, qui ne contient plus que Streptocara, devient donc celle des Streptocarinæ. Nous partageons entièrement l'avis de Osche sur les affinités de Seuratia et de Rusguniella, mais nous verrons plus bas que l'ensemble de ces genres nous semble devoir être rattaché à Streptocara, ce qui permet de conserver le cadre primitif offert par la sous-famille des Seuratiinæ. D'autres modifications secondaires ont été vivement critiquées par Sobolev (1957).

Il pense en particulier que le nouveau genre Stammerinema proposé par Osche est synonyme de Skrjabinoclava, et il est vrai que les deux genres sont proches et que la nature de l'hôte n'est pas un argument suffisant pour isoler le genre. Mais $S$. soricis présente une énorme dilatation de la région œsophagienne (cf. dessins de Tiner, 1951), qui l'apparente un peu aux Tétraméridés ; cet élément nous semble suffisamment original et important pour justifier le genre Stammerinema.

Osche a proposé la mise en synonymie de Dispharynx et de Synhimantus. Nous ne pensons pas, comme Sobolev, que la structure fine des cordons est fondamentalement différente dans les deux genres. Sur coupes à main levée, nous avons pu vérifier, comme il était prévisible, que les Synhimantus primitifs (sous-genre Synhimantus) se rapprochent du type Dispharynx, alors que les Synhimantus évolués (sous-genre Desportesius) se rapprochent du genre Cosmocephalus. Il est possible de constater également que la partie antérieure du cordon a une structure plus simple et plus symétrique que la partie postérieure. Nous préférons considérer ici le genre Dispharynx comme sous-genre de Synhimantus, car il est vrai que les limites entre les deux genres sont imprécises, mais le genre Dispharynx marque cependant une étape évolutive plus primitive que le genre Synhimantus et conserve par là son intérêt.

\section{III. - MODIFICATIONS PROPOSEES}

Nous avons cherché à montrer récemment qu'il existe tous les intermédiaires entre le genre Rusguniella et le genre Seuratia, et avons proposé d'admettre l'existence d'une série évolutive distincte des autres Acuaridés. Cette lignée renferme toutes les espèces à cordons courts limités à la région cervicale, et évolue comme chez les Acuaridés à cordons allongés, par la complexité croissante des 
cordons et par l'acquisition d'épines. Nous avons ainsi par ordre de complexité croissante les genres : Rusguniella, Aviculariella, Proyseria, Paryseria et Seuratia.

\section{Larve de Paryseria heardi.}

Une larve étudiée récemment par Mawson (1953) apporte une solide confirmation à l'individualité du phylum Rusguniella-Seuratia établi précédemment, puisqu'elle confirme le mode évolutif qui avait été proposé pour interpréter le groupe.

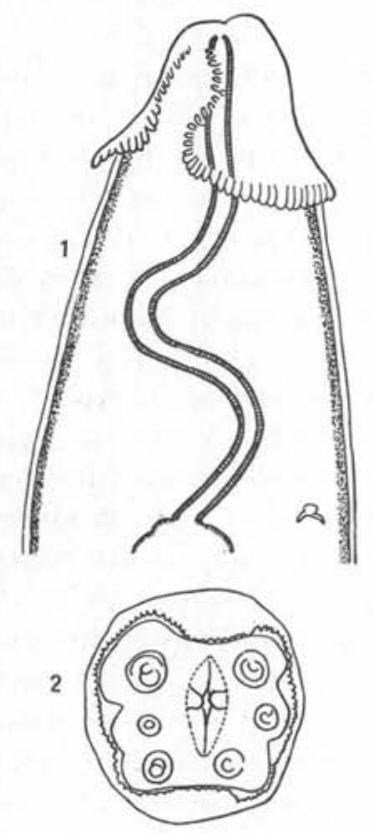

Fig. A

1. Paryseria heardi (Mawson 1953). Larve parasite d'Oceanites oceanicus. Matériel $n^{\circ} \mathrm{H} /$ / 49/706, aimablement communiqué par Mrs Thomas-Mawson. - 2. Streptocara crassicauda (Creplin 1829). Femelle parasite d'Anas boschas L., de Richelieu (Indre-et-Loire) le 22 août 1944. Collection et détermination C. Desportes. Vue apicale montrant que l'ornementation céphalique monte légèrement sur les axes médians, mais n'atteint pas les angles labiaux.

Les spécimens adultes de cette espèce ont tous les caractères du genre Paryseria tel que nous l'avons récemment redéfini, et la larve qui accompagne les adultes présente les caractères suivants : « Cervical papillae minute and bicuspid... The collar is not festooned as in adult members of the genus, but lies straight across the body except for slight indentation dorsally and ventrally. Its posterior border is not toothed, but appears as if finely scalloped, these small folds being far more numerous than the forty teeth found in the adult collar. » Mrs. Thomas-Mawson, que nous remercions vivement à ce propos, a eu la gentillesse de nous confier cette si intéressante larve. 
La convexité en avant du collier sur les axes médians est plus marquée que dans le genre Streptocara. Dans l'état actuel, la larve est trop aplatie pour pouvoir être étudiée en vue strictement médiane et nous n'avons pas pu observer personnellement si la denticulation naît des angles labiaux comme chez l'adulte, ou si elle forme un collier simplement indenté sur les axes médians comme dans le genre Streptocara.

De toute façon, cette larve forme un nouvel exemple de récapitulation ontogénique. Elle montre bien que les dents deviennent de plus en plus grandes et de moins en moins nombreuses, et, d'après l'observation primitive de Mawson, elle permet de supposer qu'il existe des formes intermédiaires entre les cordons qui naissent aux angles labiaux (phylum allant de Rusguniella à Seuratia) et les cordons formant un collier péricervical (genre Streptocara).

Genre Stegophorus.

Nous ne pensons pas que le genre puisse être confondu avec les Paryseria comme l'ont indiqué Johnston et Mawson (1945), ne serait-ce que par la structure très particulière du grand spicule.

La structure céphalique est plus difficile à interpréter, car, d'après la figure 6 de Wehr (1934), on pourrait croire qu'elle diffère peu de celle de Paryseria, alors que, d'après la figure 7, il semble exister un collier denticulé qui se continue d'une face latérale à l'autre, sans monter jusqu'à l'angle labial, ce qui la rapprocherait de Streptocara.

En conclusion, le genre Stegophorus doit être classé parmi les Seuratiinæ, mais il est actuellement difficile de savoir s'il ne diffère des Paryseria que par la structure du spicule, ou si, au contraire, l'anatomie céphalique permet de le considérer comme intermédiaire entre le genre Streptocara et le groupe Rusguniella-Seuratia.

Genre Streptocara.

La larve de Paryseria heardi, ainsi que l'adulte de Stegophorus stella-polaris ne nous permettent donc pas de conclure formellement à une filiation directe entre l'ornementation céphalique du type Streptocara et celle des genres où l'ornementation céphalique nait au niveau des angles labiaux, mais, même si l'on refuse d'admettre cette filiation directe, nous pensons que le rapprochement entre Streptocara et Rusguniella, déjà admis par les auteurs russes, peut être conservé (1).

(1) De toutes façons, il est impossible de considérer les Streptocara actuels comme les ancêtres directs des autres genres, car si les cordons paraissent bien revêtir le type le plus primitif, les très grosses diérides sont au contraire d'un type spécialisé. 
Admettant ainsi dans la même sous-famille (mais aux deux positions extrêmes) les genres Streptocara et Seuratia, il y a très peu de modifications à introduire dans la systématique de Chitwood et Wehr.

\section{IV. - ESSAI DE CLASSIFICATION}

\section{Famille des ACUARIIDAE Seurat 1913}

DÉFinition: Spiruroidea. Bouche allongée dorso-ventralement. Pseudo-lèvres très grandes envahissant toute la surface céphalique et souvent terminées par une petite pièce triangulaire saillante en avant. Lèvres dorsale et ventrale absentes. Stoma cylindrique, souvent très allongé. Papilles céphaliques du cycle interne absentes. Cycle externe réduit à quatre papilles insérées sur les pseudolèvres. Ornementation cuticulaire céphalique présente (cordons, collerettes, boucliers ou lames cuticulaires). Ailes caudales généralement bien développées. Spicules généralement inégaux et dissemblables. Gubernaculum généralement absent. Vulve pré-équatoriale ou post-équatoriale. Ovipares. La grande majorité des espèces est parasite du gésier des Oiseaux.

La famille comprend trois sous-familles :

\section{A) Acuariinae Railliet, Henry et Sisoff 1912.}

DÉFINITION : Acuariidær, avec pièce triangulaire saillante en avant de chaque pseudo-lèvre toujours présente ; ornementation céphalique formée de cordons très allongés, généralement étendus longuement en arrière des pseudo-lèvres sur la région cervicale, ou plus rarement formant un dessin complexe sur la région céphalique (sauf dans le genre primitif Paracuaria où les cordons forment quatre bandes, simples et très courtes).

\section{B) Seuratiinae Chitwood et Wehr 1932.}

DÉFINITION : Acuariidæ, avec pièce triangulaire saillante en avant de chaque pseudo-lèvre toujours présente. Ornementation céphalique formée d'une collerette ou de cordons courts, formant un dessin peu tortueux sur les pseudo-lèvres et n'atteignant pas la région cervicale (1).

(1) La limite entre la \& région céphalique » et la \& région cervicale \$ n'est généralement pas nettement limitée, mais l'on peut admettre que la liauteur de la « région céphalique » ne dépasse pas la largeur du corps. 


\section{C) Schistorophinae Travassos 1918.}

DÉFINITION : Acuariidæ, avec pièce triangulaire saillante en avant de chaque pseudo-lèvre présente ou absente. Ornementation céphalique formée par des boucliers, des écussons ou des lames cuticulaires.

\section{Tablead Dichotomeve DES Gentes (1)}

Etant donné le grand nombre de genres récemment introduits dans la systématique, nous pensons qu'il est utile de donner les tableaux dichotomiques des genres, en modifiant légèrement ceux qui ont été donnés par Osche (1955) pour les Acuariinæ et par Dollfus et Chabaud (1957) pour les Schistorophinæ.

\section{Sous-famille des Acuariinae}

1- (2) Cordons très courts, ni récurrents, ni anastomosés ..... ........................ Paracuaria Rao 1951.

2- (1) Cordons longs.

3- (8) Cordons ni récurrents, ni anastomosés.

4- (5) Crête cuticulaire ventrale précloacale chez le mâle. Touffe d'épines caudales chez la femelle . . . . . . . . . . . . ................ Skrjabinocerca Schikhobalowa 1930.

5- (4) Pas de crête cuticulaire chez le mâle, ni de touffe d'épines chez la femelle.

6- (7) Spicules égaux ............. Acuaria Bremser 1811.

7- (6) Spicules inégaux ......... Cheilospirura Diesing 1861.

8- (3) Cordons anastomosés ou récurrents.

A. Cordons anastomosés, non récurrents ou peu récurrents, sans sinuosités antérieures. Pas de collerette

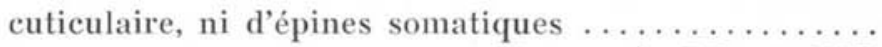
Syncuaria Gilbert 1927.

a) Cordons céphaliques simples non spinuleux. Pas de cordons supplémentaires sur les lignes latérales .. ..... Sous-genre Syncuaria (Gilbert 1927 genre).

b) Cordons céphaliques spinulés. Lignes latérales en arrière des diérides, ornées de cordons ......... Sous-genre Chordocephalurus (Alegret 1941 genre)

(1) Le genre Koriakinema Oschmarin 1949 n'est pas classé. 


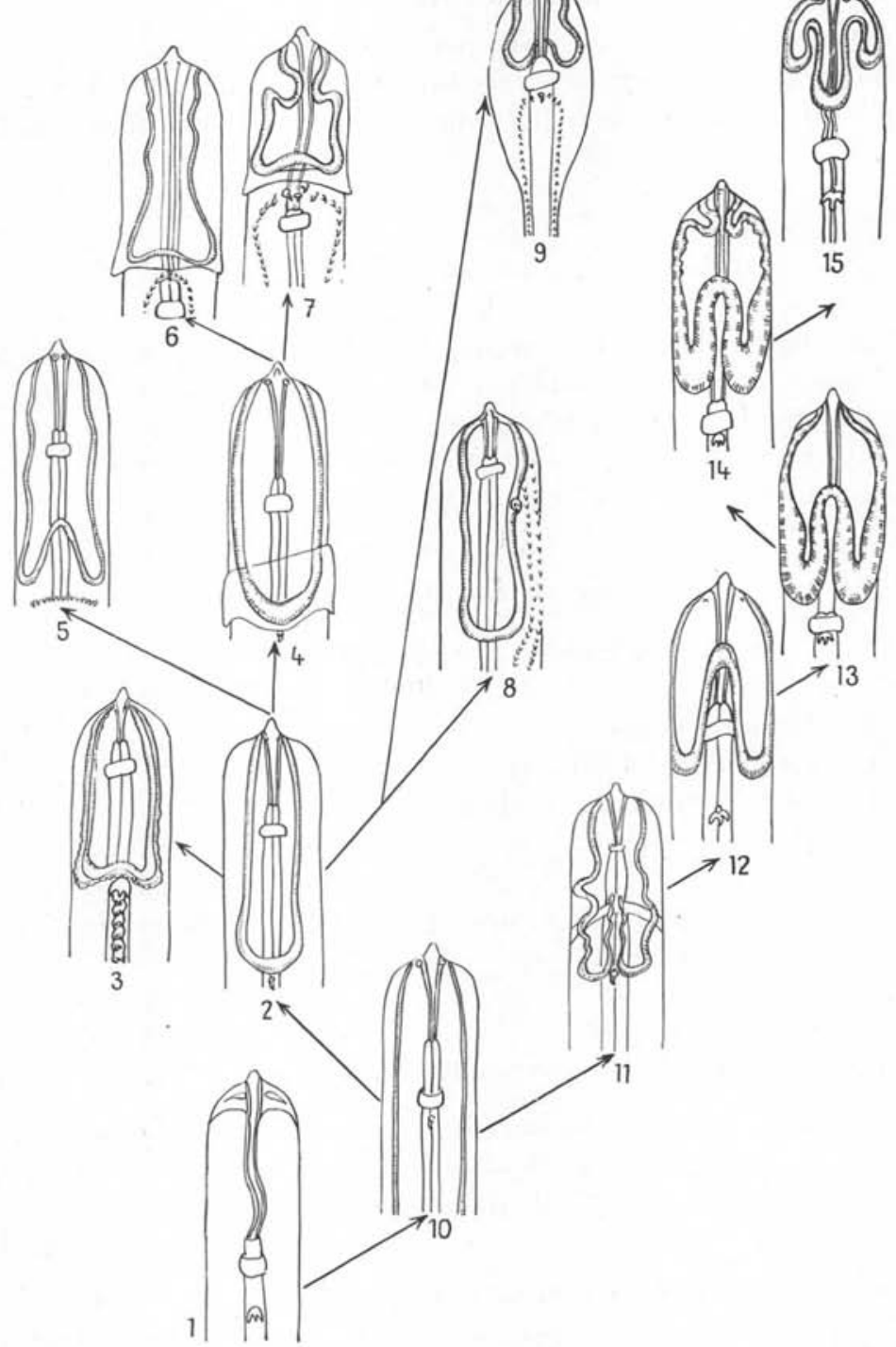

Fí. B. - Hypothèses sur la filiation des ornementations cuticulaires dans la sous-famille des Acuariine

1. Paracuaria adapté de K. Rao 1951.

2. Syncuaria (Syncuaria) adapté de Cram 1927.

3. Syncuaria (Chordocephalurus) adapté de Sobolev 1949.

4. Chevreuxia adapté de Seurat 1918.

5. Pectinospirura adapté de Wehr 1933. 
ou Sous-genre Skrjabinocara (Kuraschvili 1941 genre ?) (1).

B. Cordons anastomosés, non récurrents, sans sinuosités antérieures. Pas d'épines somatiques. Collerette cuticulaire bien développée au niveau de l'extrémité postérieure des cordons ........ Chevreuxia Seurat 1918.

C. Cordons anastomosés, légèrement récurrents, sans sinuosités antérieures. Diérides transformées en une ligne transversale d'épines en arrière des cordons .... Pectinospirura Wehr 1933.

D. Cordons anastomosés, non récurrents, avec ou sans sinuosités antérieures. Collerette cuticulaire présente. En arrière des cordons, diéride simple suivie de deux lignes d'épines sur chaque face latérale ............ .................. Skrjabinoclava Sobolev 1941.

E. Cordons anastomosés, non récurrents, sans sinuosités antérieures. Pas de collerette cuticulaire. Les cordons ne sont pas placés symétriquement sur chaque face latérale, mais sont légèrement repoussés ventralement, car les diérides sont dorsales aux cordons. Deux files d'épines suivent les diérides sur chaque face latérale .. .................. Echinuria Soloviev 1912.

F. Cordons anastomosẻs ou non anastomosés, récurrents, sans sinuosités antérieures. Collerette cuticulaire suivie de deux lignes d'épines sur chaque face latérale. Extré-

(1) Chordocephalurus date du mois d'août 1941 , mais ne connaissant pas la date exacte de parution de Skrjabinocara, nous ne savons pas quel est le nom qui a priorité.

6. Skrjabinoclava à cordons non contournés adapté de Sobolev 1952 .

7. Skrjabinoclava à cordons contournés adapté de Desportes (dessins faune de France).

8. Echinuria adapté de Desportes (dessins faune de France).

9. Stammerinema adapté de Tiner 1951.

10. Acuaria adapté de Desportes (dessins faune de France).

11. Synhimantus (Dispharynx) adapté de Desportes (dessins faune de France).

12. Synhimantus (Synhimantus) adapté de Desportes 1947 .

13. Synhimantus (Desportesius) adapté de Chabaud et Campana 1949 .

14. Cosmocephalus adapté de Desportes (dessins faune de France).

15. Sexansocara adapté de Sobolev et Soudarikov 1939 (in Skrjabin et coll. $1949)$. 
mité antérieure du corps fortement dilatée ......... .................. Stammerinema Osche 1955.

G. Cordons anastomosés ou non anastomosés, fortement récurrents. Collerette cuticulaire absente. Pas d'épines somatiques.

a) Cordons sans sinuosités antérieures ............ ..... Synhimantus Railliet, Henry et Sisoff 1912.

$\alpha$ Cordons non anastomosés $\ldots \ldots \ldots \ldots \ldots \ldots$. Sous-genre Dispharynx (Railliet, Henry et Sisoff 1912, genre).

$\beta$ Cordons anastomosés. Vulve dans la partie moyenne du corps. Didelphe ............... Sous-genre Synhimantus (Railliet, Henry et Sisoff 1912, genre).

$\gamma$ Cordons anastomosés, très élargis postérieurement. Vulve postérieure. Monodelphe ......... Sous-genre Desportesius Chabaud et Campana 1949.

b) Cordons avec sinuosités antérieures.

a Récurrence des cordons sur les faces latérales, formée d'une courbe simple convexe en avant . . Cosmocephalus Molin 1858.

$\beta$ Récurrence des cordons sur les faces latérales, formée par deux courbes convexes en avant, séparées par une courbe concave en avant ......... ..... Sexansocara Sobolev et Soudarikov 1939.

\section{Sous-Famille des Seuratiinae}

1- (2) Moitié postérieure du grand spicule très contournée ..... ........................ Stegophorus Wehr 1934.

2- (1) Grand spicule de structure normale.

3- (4) Cordons cuticulaires ne prenant pas naissance aux commissures des lèvres buccales, mais formant une collerette continue entourant la région céphalique $\ldots \ldots \ldots \ldots \ldots$. Streptocara Railliet, Henry et Sisoff 1912.

4- (3) Cordons cuticulaires prenant naissance aux commissures des lèvres buccales.

5- (8) Cordons cuticulaires dont le bord postérieur ne se détache pas de la cuticule sous-jacente pour former une collerette denticulée. Diérides simples. 


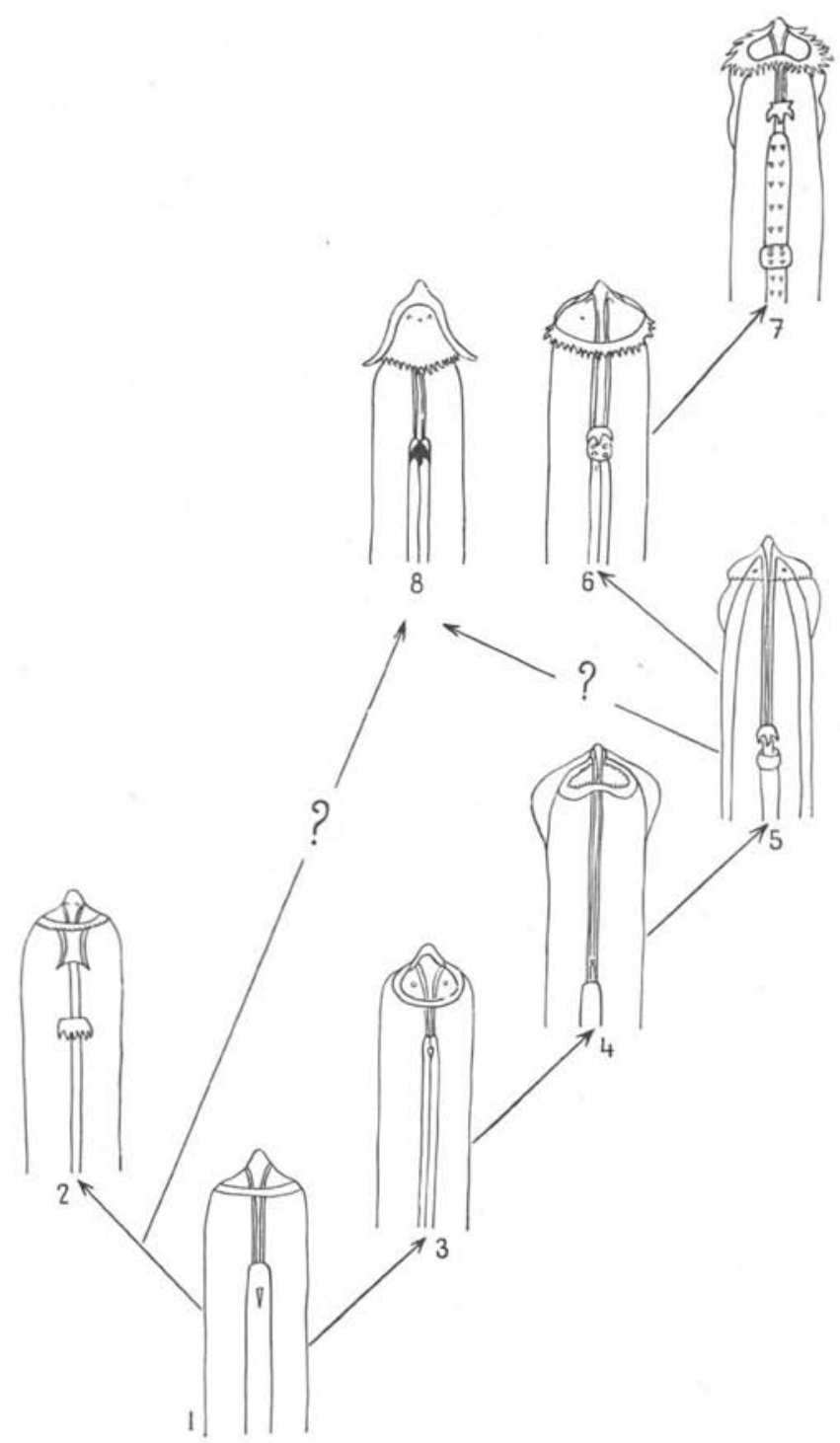

Fig. C. - Hypothèses sur la filiation des ornementations cuticulaires dans la sous-famille des Seuratiine

1. Seuratiinæ primitif hypothétique ; cordon simple, transversal, n'atteignant pas les angles labiaux.

2. Streptocara, dessin original schématisé.

3. Rusguniella adapté de Seurat 1919.

4. Aviculariella adapté de Wehr 1931.

5. Proyseria adapté de Chabaud 1953.

6. Paryseria adapté de Petter 1959.

7. Senratia adapté de Seurat 1916.

8. Stegophorus adapté de Wehr 1934. 

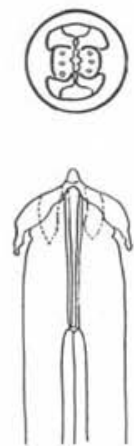

1
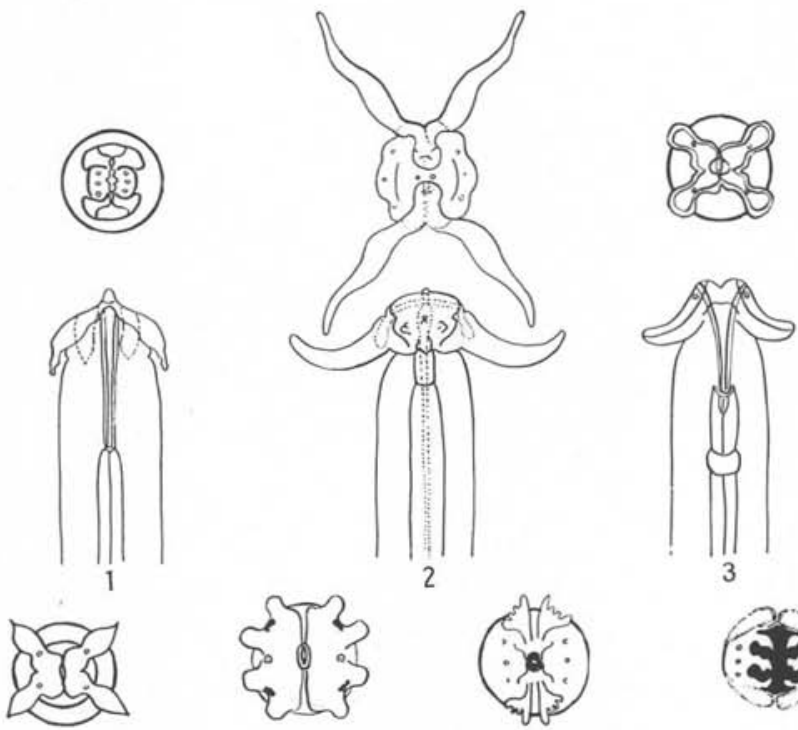

3
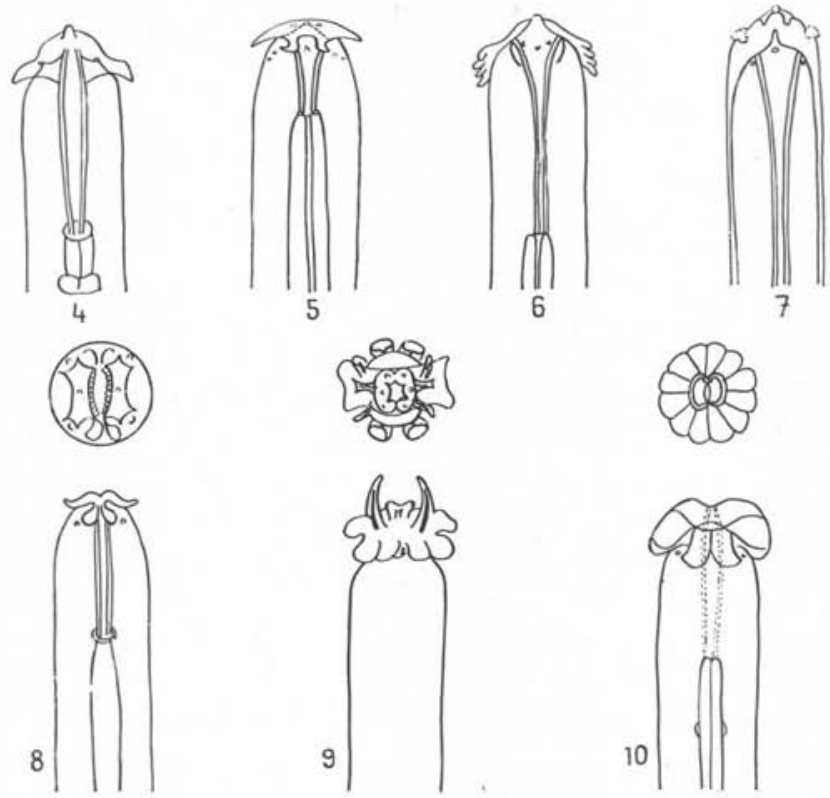

F1G. D. - Schéma des structures céphaliques dans la sous-famille des Schistorophina

1. Schistorophus à cornes courtes adapté de Wehr 1934 .

2. Schistorophus à cornes longues adapté de Li 1934 .

3. Sciadiocara adapté de Sobolev 1949 (in Skrjabin et coll. 1949). 
6- (7) Cordons cuticulaires étendus sur les faces latérales en forme de croissants dont les bords sont lisses .......... Rusguniella Seurat 1919.

7- (6) Cordons cuticulaires dessinant sur les faces latérales un triangle ; bord antérieur des cordons denticulé ......... ....................... Aviculariella Wehr 1931.

8- (5) Cordons cuticulaires dont le bord postérieur se détache de la cuticule sous-jacente pour former une collerette denticulée ; diérides tricuspides.

9-(10) Les cordons cuticulaires forment les marges de deux lames latérales recouvrant la tête. Ces marges ont des denticulations fines et nombreuses ....... Proyseria Petter 1958.

10- (9) Les cordons cuticulaires ne forment pas les marges de deux lames latérales recouvrant la tête, mais forment une collerette entourant la région céphalique.

11-(12) Pas de rangées d'épines sur les faces latérales. Cordons non récurrents sur les faces latérales, avec dents assez fines et nombreuses ......... Paryseria Johnston 1938.

12-(11) Double rangée d'épines sur chaque face latérale. Cordons légèrement récurrents sur les faces latérales, avec dents grosses et peu nombreuses ..... Seuratia Skrjabin 1916.

\section{Sous-Famille des Schistorophinae}

1- (2) Tête ornée seulement de 4 lames ou appendices, simples ou divisés à leur extrémité distale, qui naissent aux angles labiaux. Pseudo-lèvres terminées généralement par une pointe apicale.

A. 4 cornes cylindriques, longues ou courtes $\ldots \ldots \ldots \ldots$. Schistorophus Railliet 1916 (= Quasithelazia Maplestone $=$ Antennocara Wassilkowa).

B. 4 lames arrondies ...... Sciadiocara Skrjabin 1916.

4. Viktorocara adapté de Guschanskaja 1950 (vue apicale reconstituée hypothétiquement).

5. Ancyracanthopsis à lames peu divisées adapté de Li 1934 .

6. Ancyracanthopsis à lames très divisées adapté de Clapham 1945.

7. Schistogendra adapté de Chabaud et Rousselot 1956.

8. Viguiera adapté de Chabaud 1957 .

9. Serticeps adapté de Drasche 1882 .

10. Torquatella adapté de Drasche 1882 .

Ann. de Parasitologie, t. XXXIII, No $3,1959$. 
C. 4 lames pointues ... Viktorocara Guschanskaja 1950 .

D. 4 lames subdivisées en dents ou en lanières à l'apex .. .................. Ancyracanthopsis Diesing 1861

(= Skrjabinobronema Guschanskaja $1950=$ Parahistiocephalus Belopolskaja 1953).

2- (1) Ornementation céphalique comportant également des écussons qui intéressent l'axe médian et qui débordent souvent sur la région cervicale. Pointe apicale des pseudo-lèvres souvent absente.

3- (4) Bord libre des pseudo-lèvres profondément découpé .... ........... Schistogendra Chabaud et Rousselot 1956.

4- (3) Bord libre des pseudo-lèvres entier.

5- (6) Bouche entourée de 6 lames, 2 lames latérales et 4 lames ou écussons submédians.

A. Pointe apicale des pseudo-lèvres présente $\ldots \ldots \ldots \ldots$ ..................... Krusadia Sanwal 1952.

B. Pointe apicale des pseudo-lèvres absente .......... ........................ Viguiera Seurat 1913.

6- (5) Tête ornée d'écussons cuticulaires plus nombreux.

A. Les écussons cuticulaires s'étendent sur les faces médianes et latérales en une seule rangée..$\ldots \ldots \ldots \ldots$ Serticeps Railliet 1916.

B. Les écussons cuticulaires s'étendent sur les faces médianes et latérales en plusieurs rangées ........... Torquatella Yorke et Maplestone 1926.

\section{RÉSUMÉ}

Dans la famille des Acuarïdx, la morphologie des adultes, la morphologie des larves et la vitesse du développement chez l'hôte intermédiaire fournissent des documents variés, qui se recoupent les uns les autres et permettent d'établir une phylogénie qui semble relativement très précise. La larve de Paryseria heardi, découverte par Mawson, donne de nouveaux éléments intéressants à ce point de vue.

En empruntant quelques modifications parmi celles qui apparaissent dans les travaux récents de Sobolev et de Osche, il est possible de s'éloigner très peu de la systématique établie par Chitwood et Wehr et d'obtenir une classification qui semble en accord avec les données phylogéniques. 


\section{Nous proposons :}

a) De supprimer le genre Yseria dont l'espèce type est un Streptocara et de reclasser parmi les Schistorophinæ les deux espèces : Ancyracanthopsis coronata (Molin 1860) nov. comb. et A. quadripartita (Clapham 1945) nov. comb.

b) De reprendre la sous-famille des Seuratiinæ en amendant sa définition pour y admettre, en même temps que Streptocara et Seuratia, les genres Rusguniella, Aviculariella, Proyseria, Paryseria et Stegophorus. (La structure céphalique de ce dernier genre prêtant encore à discussion, nous ne basons actuellement son individualité que sur la structure particulière du grand spicule).

c) Les genres Dispharynx et Chordocephalurus (ou Skrjabinocerca ?) sont conservés, mais seulement au rang de sous-genres, respectivement de Synhimantus et de Syncuaria.

d) La synonymie de Stammerinema et de Skrjabinoclava est rejetée.

Des tableaux dichotomiques conduisant aux genres sont proposés pour les trois sous-familles.

\section{BIBLIOGRAPHIE}

Alegret (M. J.), 1941. - Contribucion al conocimiento de los vermes parasitos de Phalacrocorax auritus floridanus. Thèse (Univ. Habana), 32 pp., Havane (août).

Chabaud (A. G.), 1949. - A propos du cycle évolutif d'un Synhimantus (Nematoda-Acuarida), recherche des lois qui régissent la vitesse du développement suivant la place zoologique du parasite et la biologie de l'hôte intermédiaire. Bull. Soc. Zool. France, LXXIV, 342-345.

- 1953. - Sur un Nématode Acuariidæ parasite du Martin-Pêcheur Alcedo atthis (L.). Ann. Parasit., XXVIII, 365-371.

- 1954. - Sur le cycle évolutif des Spirurides et de Nématodes ayant une biologie comparable. Valeur systématique des caractères biologiques. Ann. Parasit., XXIX, 42-88, 206-249, 359-425.

- 1957. - Nématodes parasites d'Oiseaux en Tunisie. Collection C. Vermeil. Arch. Inst. Pasteur Tunis, XXXIV, 155-166.

et Campana (Y.), 1949. - A propos d'une variété nouvelle de Synhimantus equispiculatus Wu et Liu 1943. Création d'un nouveau sous-genre (Desportesius) N. Subgen. Ann. Parasit., XXIV, n ${ }^{\circ}$ 1-2, 77-92.

et Rousselor (R.), 1956. - Description d'un nouvel Acuariide d'Afrique Equatoriale: Schistogendra incisa n.gen., n. sp. Ann. Parasit,. XXXI, 242-247.

Chitwood (B. G.) \& WeHr (E. E.), 1934. - The value of cephalic structures as characters in nematode classification, with special reference to the superfamily Spiruroidea. Zeitsch. f. Parasit., VII, 273-335 + 1 pl. 
Clapham (P. A.), 1945. - Some bird Helninths from Antigua. Journ. Helminth., XXI, 93-99.

Свам (E. B.), 1927. - Bird parasites of the nematode suborders Strongylata, Ascaridata, and Spirurata. U.S. Nat. Museum, Bull. $\mathrm{n}^{\circ} 140$, xviI + 465 pp.

Despontes (C.). - Dessins inédits exécutés pour la préparation des Nématodes de la faune de France.

Dollfus (R.-P.) et Chabaud (A.-G.), 1957. - Phénomènes de convergence chez les Spirurides, en particulier dans les sous-familles Habronematinæ Chitwood et Wehr 1932 et Schistorophine L. Travassos 1918; leur importance pour une classification naturelle des Spirurides (Nematoda). Bull. Soc. Zool. France, LXXXII, 88-102.

Drasche (R. von), 1882. - Revision der in der Nematoden-Sammlung des k.k. zoologischen Hofcabinets befindlichen Original-Exemplare Diesing's und Molin's. Verhandl. d. k.k. zool. bot. Gesellsch. in Wien (1882), XXXIII, 107-118, pl. 3-5, 193-218.

Gedoelst (L.), 1919. - Le genre Histiocephalus et les espèces qui y ont été rapportées. C.R. Soc. Biol., LXXIII, 901-903.

- et LIÉGEoIs (E.), 1922. - Note sur le Streptocara pectinifera (Neumann). C.R. Soc. Biol., LXXXVII, 1237-1239.

GiLbert (L. I.), 1927. - Zur Charakteristik zweier Vogel-nematoden des Westens der Union S.S.R. Sborn. Rabot Gelmintol. Posv. K.I. Skrjabin, 54-61 (en russe, résumé en allemand).

Guschanskaja (L. K.), 1950. - Nouveaux Spirurides d'Oiseaux. Trudi Gelmint. Labor., IV, 40-52 (en russe).

1951. - Modifications dans la classification des Nématodes des familles Acuariidæe et Histiocephalidx. Trudi Gelmint. Labor., V, 90-92 (en russe),

Johnston (T. H.) \& Mawson (P. M.), 1945. - Parasitic Nematodes. B.A.N.Z. Antaret. Res. Exp., 1929-1931. Reports, Ser. B, V, 159, 51 fig.

Kurachvili (B. E.), 1941. - Etude sur la faune helminthologique des Oiseaux de Géorgie. Trav. Inst. Zool. A.N., S.S.R. de Géorgie, IV, 53-100 (en russe).

LI (H. C.), 1934. - Report on a collection of Parasitic Nematodes, mainly from North China, Part II. Spiruroidea. Trans. Amer. Micros. Soc., LIII, 174195, pl. 13-16.

Mawson (P. M.), 1953. - Parasitic Nematoda collected by the Australian National Antartic, Research expedition : Heard Island and Macquarie Island, 1948-1951. Parasit., XLIII, 292-297.

Osche (G.), 1955. - Bau, Entwicklung und systematische Bedeutung der Cordons der Acuariide (Nematoda) am Beispiel von Stammerinema soricis (Tiner 1951) gen. nov. Z. f. Parasit., Bd. 17, 73-92.

Petter (A. J.), 1959. - Redeseription de Paryseria adelia Johnston 1938. Remarques sur la série Rusguniella, Aviculariella, Proyseria (gen. nov.), Paryseria, Seuratia. Ann. Parasit., XXX1V, 322-330.

Railliet (A.), Henry (A.) et Sisoff (P.), 1912. - Sur les affinités des Dispharages (Acuaria Bremser), Nématodes parasites des Oiseaux. C.R. Soc. Biol., LXXIII, 622-624.

Rao (N. S. K.), 1951. - Paracuria macdonaldi n.g. n. sp. (Acuariinæ) from the sea gull (Larus argentus). Canad. J. Zool., XXIX, 167-172. 
SANWAL (K. C.), 1952. - On a new avian nematode, Krusadia indica n.g., n. sp. (sub. fam. Schistorophina Travassos 1918) from the Jungle Nightjar (Caprimulgus indicus. Indian J. Helmin., IV, 54-60.

Sснікновацоwa (N.), 1930. - On a new genus of the Nematode fam. Acuarida Seurat, 1913. J. Parasit., XVI, 220-223.

Seurat (L. G.), 1916. - Sur un nouveau Dispharage des Palmipèdes. C.R. Soc. Biol., LXXIX, 785.

- 1918. - Sur le Dispharage de l'Echasse. Bull. Soc. Hist. Nat. de l'Afrique du Nord, IX, 106-109.

- 1919. - Contributions nouvelles à l'étude des formes larvaires des Nématodes parasites hétéroxènes. Bull. Biol. France et Belgique, LII, 344-378.

SKRJABIN (K. I.), 1941. - On the rearrangement of the taxonomy of nematodes of the Acuariide and Ancyranthide families. C.R. (Dokl.). Acad. Sc. U.R.S.S., XXX, 470-473.

- Schiкhobalova (N. P.) \& Sobolev (A. A.), 1949. - Opredelitel parazititcheskich. Nematod., I, Spirurides et Filaires, 521 (en russe).

Soвolev (A. A.), 1952. - Skrjabinoclava longifuniculata n. sp., nouveau Nématode d'Oiseaux. Trudi Gelmint. Labor., VI, 293-295 (en russe).

1957. - Evaluation of the application of comparative ontogenic method in the systematics of Spirurats (Nematoda, Spirurata). Zoologitcheski Journ., XXXVI, 1304-1311 (en russe, résumé en anglais).

TINer (J. D.), 1951. - Dispharynx soricis n. sp. (Nematoda : Acuariida) from the Shrew Sorex obscurus alascensis, and associated Host Pathology. Proc. Helmint. Soc. Washington, XVIII, 64-70.

WeHr (E. E.), 1931. - Description of a new genus and species of Nematode worm occurring in the northwestern belted Kingfisher with a key to the genera of the subfamily Acuariina. Proc. U.S. Nac. Mus., LXXIX, 1-4.

- 1933. - Descriptions of two new parasitic nematodes from birds. $J$. Washington Acad. Sc., XXIII, 391-396.

1934. - Descriptions of three bird nematodes, including a new genus and a new species. J. Washington Acad. Sc., XXIV, 341-347.

Williams (O. L.), 1929. - Revision of the Nematode genus Rusguniella Seurat. With a description of a new central american species. Univ. California Public. Zool., XXXIII, 1-12.

YoRke (W.) \& MAPLestone (P. A.), 1926. - The Nematode parasites of Vertebrates, $\mathrm{x}+536$ pp., Londres. 